\title{
Beam Blanking Studies in Environmental Scanning Electron Microscopy
}

\author{
H. Dobberstein*, N. J. Purser*, T. W. Fairhead*, D. J. Stokes*, and R.W. Knowles** \\ *Department of Physics, Cavendish Laboratory, University of Cambridge, Cambridge, CB3 0HE, \\ UK. \\ **FEI Company, 5350 NE Dawson Creek Drive, Hillsboro, OR 97124.
}

Blanking or modulating of the primary electron beam has become of great interest in the electron microscopy community for physical applications, including electron beam lithography [1] or electron beam-induced current (EBIC) [2], as well as for decreasing beam damages in biological or polymeric specimens during electron irradiation.

Our beam blanking system was manufactured by FEI Company, and fitted to a FEI XL 30 ESEM (field-emission). Measurements have shown that the beam blanker has a maximum usable repetition rate at $10 \mathrm{MHz}$, a start/end blank delay in the range of 40ns, and a blanking on/off rise time of 10ns. We designed the beam blanker driver that it can be triggered by both the CBLK signal from the SEM and an external pulse/signal generator. The output of the pulse generator has been linked to an oscilloscope, to help setting up the waveform frequency and/or pulse width. Additionally, we connected the SEM CBLK signal to the trigger input of the pulse generator in order to synchronize the signal of the pulse generator with the SEM scanning unit. The setup allowed us to blank the primary electron beam $(0 \%, 5-95 \%, 100 \%)$ during an active line scan, and to block the beam completely when the scan returns to the start position of new the line scan. A block diagram of the experimental setup is given in Figure 1.

Beam blanking experiments have shown that it is necessary to use a beam blanking frequency, which is at least 10 times smaller than the time the beam needs to scan along the width of a pixel to avoid band artifacts. In our case we used the "default" setting for a single scan, which is $20 \mathrm{~ms}$ per line scan, and 484 lines per frame. As the saved images had a resolution of $645 \times 484$ pixels, the average scanning time per pixel (in a line scan) was given as $31.5 \mu \mathrm{s}$ or $32 \mathrm{kHz}$. An SEM micrograph, where $50 \%$ of the primary beam was blanked at a pulse frequency of $32 \mathrm{kHz}$, is shown in Figure 2a. The band lines in the image were caused by a small mismatch between the beam blanking frequency and the actual scanning frequency. The line effects disappeared and no other artifacts were visible, when the beam blanking frequency was increased to $320 \mathrm{kHz}$ (Figure 2b). As expected the micrograph appears darker than the image obtained without beam blanking (Figure $2 \mathrm{c}$ ).

In this presentation, we will discuss how the degree of beam blanking affects the quality of STEM micrographs in high vacuum mode. Then, we show how high vacuum results will alter by varying ESEM operating conditions, including beam energy $(\mathrm{kV})$, beam current, working distance, gas pressure, and gas type. The obtained EM images have been analyzed using the free software Scion Image Beta 4.02 (Scion Cooperation) and the plug-in macro SMART, which is written by D. C. Joy and can be downloaded from his webpage [3]. The software package employs a Fast Fourier transform to generate a diffractogram (two-dimensional power spectrum) of the image, which allows a quantitative analysis of important electron microcopy parameters, such as, the probe size, resolution, and signal to noise ratio. 
Finally, beam blanking results of Poly(methyl-methacrylate), PMMA, a polymer that degradation by electron irradiation in SEMs has been widely studied, will be presented [4,5]. We will demonstrate that by using the beam blanker the beam damage can be reduced significantly $(>80 \%)$ without compromising the image quality.

\section{References}

[1] S. W Park, et al., Jpn. J. Appl. Phys. 26 (1987) L44.

[2] S. A. L. Foulds, et al., Inst. Phys. Conf. Ser. 153 (1997) 61.

[3] D. C. Joy, et al., Proc. SPIE 3998 (2000) 108; http://web.utk.edu/ srcut.

[4] L. C. Sawyer, et al, Polymer Microscopy, 2nd ed., Chapman \& Hall, London (1996).

[5] N. Grassie, et al., Polymer Degradation and Stabilisation, Cambridge University Press, Cambridge (1988).

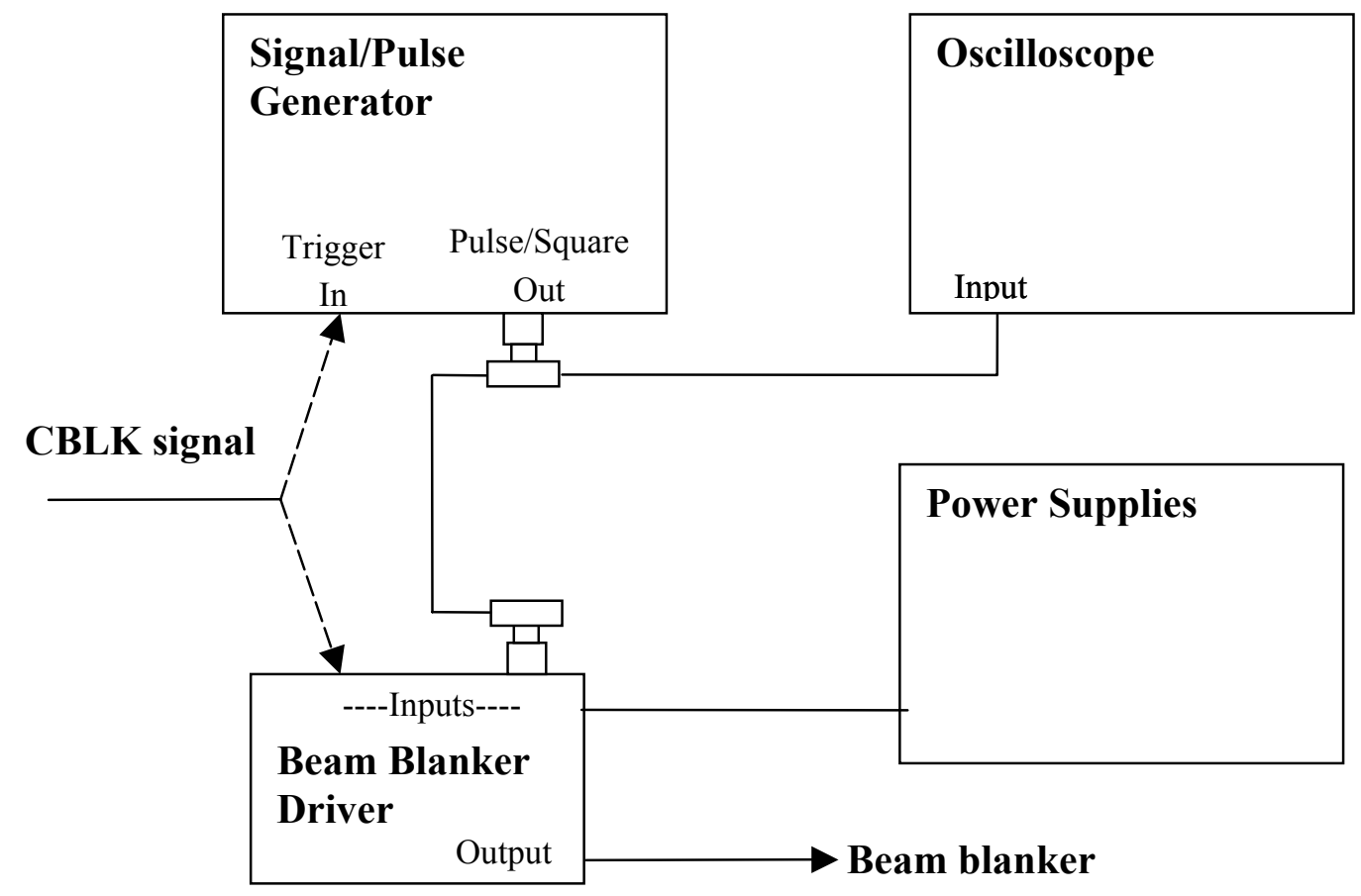

FIG. 1. Interconnection diagram showing the experimental setup of the beam blanking device.
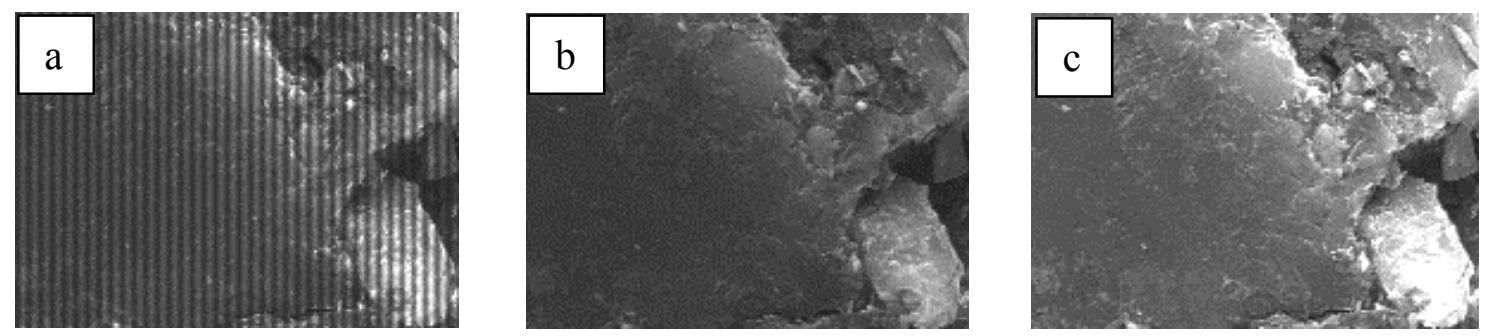

FIG. 2. SEM micrographs of gold on carbon, where $50 \%$ of the primary electrons were blanked at a frequency of (a) $32 \mathrm{kHz}$ (close to the scanning frequency), and (b) $320 \mathrm{kHz}$. Bands in (a) are due to frequency mismatch. Image (c) shows the gold layer without beam blanking. 